\title{
Estimation des élasticités de demande des produits alimentaires au Togo
}

Estimation of elasticities of demand for food products in Togo

\section{Tomgouani Lanié}

\section{(2) OpenEdition}

1 Journals

Édition électronique

URL : http://journals.openedition.org/economierurale/6942

DOI : 10.4000/economierurale.6942

ISSN : 2105-2581

Éditeur

Société Française d'Économie Rurale (SFER)

Édition imprimée

Date de publication : 30 septembre 2019

Pagination : 41-61

ISSN : 0013-0559

\section{Référence électronique}

Tomgouani Lanié, "Estimation des élasticités de demande des produits alimentaires au Togo »,

Économie rurale [En ligne], 369 | Juillet-septembre, mis en ligne le 01 janvier 2021, consulté le 17 janvier 2021. URL : http://journals.openedition.org/economierurale/6942 ; DOI : https://doi.org/ 10.4000/economierurale.6942

(c) Tous droits réservés 


\title{
Estimation des élasticités de demande des produits alimentaires au Togo
}

\author{
Tomgouani LANIE • Faculté des Sciences Économiques et de Gestion (FaSEG), \\ Département d'Économie, Université de Kara, Togo \\ lanietomgouani@gmail.com
}

Dans cet article, l'auteur a estimé les élasticités-prix et dépenses de la demande des produits alimentaires ainsi que simulé l'impact de la politique d'allègement fiscal du gouvernement sur le bien-être des ménages et la pauvreté au Togo. Les résultats montrent que les élasticités-prix compensées de la demande ont des signes attendus, c'est-à-dire négatifs, traduisant la relation inverse entre le prix et la quantité demandée d'un bien. Quant aux élasticités-revenus de la demande, elles montrent que les céréales telles que le maïs, le riz et les céréales transformées apparaissent dans la catégorie des biens de nécessité alors que le mil, le sorgho et le fonio sont des biens inférieurs. La valeur des élasticités-prix et revenus de la demande varient selon le milieu de résidence des ménages et leur statut de pauvreté. Les résultats des simulations montrent que la politique d'allègement fiscal du gouvernement semble être efficace dans l'amélioration du bienêtre des ménages et la réduction de la pauvreté.

MOTS-CLÉS : consommation des produits alimentaires, système complet de demande, données d'enquête ménages, Togo

\section{Estimation of elasticities of demand for food products in Togo}

In this article, we have estimated the price- and income-elasticity of demand for food products, and we have simulated the impact of a reduction in VAT on poverty and household welfare in Togo. As expected, results indicate that the compensated price-elasticities of demand are negative, in accordance with the inverse relationship between the price and demand of a given good. The income elasticities of demand suggest that cereals such as maize, rice, and processed cereals appear as necessity goods, whereas cereals such as millet, sorghum and fonio are inferior goods. The values of price- and income-elasticity of demand vary according to households' place of residence and their poverty status. The results of the simulations indicate that the government's policy of reducing VAT appears to be effective in improving household welfare and reducing poverty in Togo. (JEL: Q11, D12)

KEYWORDS: food consumption, complete demand system, household survey data, Togo

L es élasticités-prix et revenu de la demande qui mesurent dans quelles proportions les quantités demandées d'un bien ou d'un service varient à la suite de la variation des prix et/ou du revenu sont des paramètres clés qui permettent non seulement d'approfondir notre compréhension du comportement de consommation des agents économiques ou ménages, mais aussi d'améliorer notre capacité de prévision des résultats d'une politique économique (McKelvey, 2011 ; Tafere et al., 2010). En effet, pour une mise en œuvre efficace des réformes fiscales dans les pays en développement (PED), la connaissance des élasticités-prix et revenu de la demande est nécessaire pour prévoir les dépenses publiques qu'il convient de mobiliser et les recettes qui seront engrangées à la suite de la mise en œuvre de ces réformes (Gibson et Rozelle, 2005).

Aussi, pour évaluer l'impact des politiques de systèmes de taxes indirectes et subventions qui engendrent une variation des prix relatifs, la compréhension de la réponse des ménages aux variations 
des prix à travers leur comportement de demande est nécessaire pour déterminer dans quelle mesure ces variations constituent une contrainte ou une opportunité pour les ménages afin d'appréhender les implications des réformes fiscales sur les questions d'efficience et d'équité (Gibson, 2013; McKelvey, 2011). En effet, une élasticité-prix élevée pour un bien donné indique que ce bien est un mauvais candidat à la taxation du fait des distorsions que l'application d'une telle politique est susceptible de produire (Deaton, 1997).

Dans un contexte de changement climatique et de volatilité des prix des produits sur le marché mondial, les élasticités-prix de la demande sont les paramètres clés qui permettent de saisir quels sont les sousgroupes de la population qui sont plus affectés par les chocs de prix et surtout si certaines catégories de ménages par rapport à leur revenu peuvent avoir accès ou non à des sources alternatives de produits alimentaires.

Au niveau empirique, en l'absence d'information sur les élasticités prix et revenu de la demande, les études de l'impact des réformes fiscales ou des chocs de prix sur le bien-être et la distribution des revenus sont souvent menées en faisant l'hypothèse que les consommateurs ne répondent pas aux variations des taxes ou des prix relatifs des produits en procédant à des substitutions entre produits (Abramovsky et al., 2011 ; Banks et al., 1997) alors que l'effet des reformes sur les recettes fiscales, par exemple, dépend de la manière dont elles altèrent la demande totale des ménages et non de la manière dont elle affecte de façon intensive ou extensive les marges (Deaton, 1990). Ainsi, les premières approximations qui négligent la réponse des ménages à travers les changements dans leur structure de demande peuvent sérieusement surestimer les pertes de bien-être (Gibson et Rozelle, 2005).

Malgré cette importance des élasticitésprix et revenu de la demande dans l'évaluation et l'implémentation des politiques économiques, les études qui se sont intéressées à l'estimation des élasticitésprix et revenu de la demande des produits alimentaires en Afrique subsaharienne sont rares (Nzuma et Sarker, 2010). Au Togo, dans le but de lutter contre la cherté de la vie et réduire la pauvreté, selon la loi de finance 2017, certains produits de consommation de base tels que les pâtes alimentaires, les huiles alimentaires, le sucre, la farine de céréales, le lait manufacturé, le maïs, le sorgho, le mil, le fonio et le riz (allègement de $100 \%$ de la TVA sauf pour le riz de luxe) ont bénéficié d'un allègement fiscal de huit points sur la TVA. Dans un tel contexte, les élasticités-prix de la demande sont les paramètres clés qui permettent d'inférer sur l'efficacité d'une telle politique.

Cependant, ces élasticités-prix et revenu de la demande sont quasi inexistantes au Togo. Seule l'étude menée par Tchabletienne et al. (2010) a estimé les élasticités-prix non compensées de demande du riz local et importé à partir des séries chronologiques sur la demande et les prix du riz local et importé sur la période 1986-2006. Toutefois, cette étude est limitée à la caractérisation de la nature économique et de la typologie de seulement deux produits alimentaires : le riz local et le riz importé.

Du fait de ce déficit d'information sur la valeur des élasticités-prix de demande, Agbodji et al. (2013) dans leur analyse de l'impact de la hausse des prix des produits alimentaires de base sur le bien-être des ménages au Togo se sont limités aux approximations de court terme négligeant donc les possibilités de substitution entre produits qui constituent une stratégie des ménages à court et moyen terme pour réduire leurs pertes de bien-être lorsque les chocs de prix des produits alimentaires surviennent.

Notre étude se propose donc de combler cet écart de connaissance sur la demande 
des produits alimentaires au Togo en étendant l'analyse à l'ensemble des produits alimentaires et à partir des données d'enquête sur les conditions de vie des ménages au Togo (Quibb, 2006). Dans cette étude, les valeurs des élasticités-prix compensées et revenus de la demande de l'ensemble des produits alimentaires au Togo sont déterminées à partir du modèle Quadratic Almost Ideal Demand System de Banks et al. (1997) au niveau national, selon le milieu de résidence des ménages (urbain et rural) et leur statut de pauvreté (pauvres et non-pauvres). L'analyse est basée sur 19 produits alimentaires et 1 produit non alimentaire sous l'hypothèse de séparabilité des préférences dans les décisions de consommation des ménages.

Les résultats montrent que, dans l'ensemble, les élasticités-prix compensées de la demande ont des signes attendus, c'està-dire négatifs, indiquant que lorsque le prix d'un bien augmente, toute chose égale par ailleurs, sa demande diminue. Les élasticités-revenus de la demande indiquent que les céréales telles que le maiis, le riz et les céréales transformées sont des biens de nécessité alors que le mil, le sorgho et le fonio sont des biens inférieurs. En dehors de ces trois dernières céréales, les groupes de produits œufs, lait et produits assimilés, aliments hors ménages et autres produits alimentaires apparaissent aussi comme des biens inférieurs. Le reste des produits sont des biens normaux. La valeur des élasticités-prix et revenu de demande varient selon le milieu de résidence des ménages et leur statut de pauvreté.

À partir des informations sur ces élasticités-prix de demande, nous avons simulé l'impact de la politique d'allègement fiscal sur le bien-être des ménages et la pauvreté en nous focalisant sur les trois céréales les plus consumées au Togo (maïs, sorgho et riz). Les résultats montrent effectivement que l'impact de la baisse de la TVA est sous-estimé lorsque la réponse des ménages n'est pas prise en compte. Cette politique d'allègement fiscal a eu un impact positif sur le bien-être des ménages au niveau national et suivant les différentes catégories de ménages, et s'est traduite par une baisse de la pauvreté seulement au niveau national, parmi les ménages plus aisés et au niveau des ménages consommateurs mais cet impact est marginal.

Le reste de l'article est organisé comme suit. Nous présentons tout d'abord, la méthodologie de l'étude, puis les données et les problèmes liés à l'utilisation de ces données. Ensuite, nous exposons et discutons des problèmes économétriques liés à l'estimation du modèle de demande QUAIDS à partir des données d'enquête ménages. Les résultats des estimations des élasticités de demande sont démontrés. Enfin, les résultats des simulations sont présentés. La dernière partie conclut.

\section{Méthodologie}

L'un des modèles de référence pour l'estimation des élasticités-prix et revenu de la demande est le modèle QUAIDS parce qu'il présente l'avantage d'être assez flexible pour capter le comportement de consommation des ménages. Il provient de l'optimisation d'une fonction d'utilité indirecte $U$ décrite par :

$$
\ln U=\left\{\left[\frac{\ln x-\ln a(p)}{b(p)}\right]^{-1}+\lambda(p)\right\}^{-1}
$$

où $x$ est le revenu ou le montant total des dépenses ; $p$ est le vecteur de prix ; $a(p)$ est une fonction homogène de degré un dans les prix ; $b(p)$ et $\lambda(p)$ sont des fonctions homogènes de degré zéro dans les prix. $\ln a(p)$ et $b(p)$ sont respectivement des fonctions Translog et Cobb-Douglas :

$$
\begin{aligned}
\ln a(p) & =\alpha_{0}+\sum_{i=1}^{n} \alpha_{i} \ln p_{i} \\
& +\frac{1}{2} \sum_{i=1}^{n} \times \sum_{j=1}^{n} \gamma_{i j} \ln p_{i} \times \ln p_{j}
\end{aligned}
$$




$$
b(p)=\prod_{i=1}^{n} p_{i}^{\beta_{i}}
$$

où $i=1, \ldots, n$ représentent les produits ou groupes de produits alimentaires. La fonction $\lambda(p)$ est spécifiée comme suit :

$$
\lambda(p)=\sum_{i=1}^{n} \lambda_{i} \ln p_{i} \quad \text { avec } \quad \sum_{i=1}^{n} \lambda_{i}=0
$$

Les équations (1), (2), (3) et (4) ensemble définissent le modèle QUAIDS. En appliquant l'identité de Roy à l'équation (1), le système d'équations représentant les parts budgétaires pour un ménage $h$ et pour un produit alimentaire $i$ est exprimé comme suit :

$$
\begin{aligned}
w_{h i} & =\alpha_{i}+\sum_{j=1}^{n} \gamma_{i j} p_{j} \\
& +\beta_{i} \ln \left[\frac{x}{a(p)}\right]+\frac{\lambda_{i}}{b(p)}\left\{\ln \left[\frac{x}{a(p)}\right]\right\}^{2}
\end{aligned}
$$

où $w_{h i}(u, p)$ désigne la part du budget allouée par le ménage $h$ au produit $i, n$ est le nombre de produits alimentaires indexés par $i ; p_{i}$, le prix du bien $i$ et $\alpha_{i} ; \gamma_{i j} ; \beta_{i}$ et $\lambda_{i}$ sont des paramètres à estimer. Pour les estimations économétriques, un terme d'erreur normalement et indépendamment distribué est ajouté au modèle statistique de l'équation (5). L'hypothèse de rationalité du consommateur induit un certain nombre de propriétés du système de demande :

- Additivité : $\sum_{i=1}^{n} \alpha_{i}=1 ; \sum_{i=1}^{n} \beta_{i}=0$;

$$
\sum_{i=1}^{n} \lambda_{i}=0 ; \sum_{i=1}^{n} \gamma_{i j}=0 ; \forall i
$$

- Homogénéité : $\sum_{j=1}^{n} \gamma_{j i}=0 ; \forall i$

- Symétrie de Slustky : $\gamma_{i j}=\gamma_{j i} ; \forall i, j(8)$.

Les élasticités-prix de demande non compensées (marshalliennes) sont dérivées comme suit :

$$
E_{i p}^{u}=\frac{1}{w_{h i}} \frac{\partial w_{h i}}{\partial p_{j}}-\delta_{i j}
$$

où $\delta_{i j}$ est un symbole du Kronecker delta défini comme suit : $\delta_{i j}=1$ si $i=j$ et 0 ailleurs.

En utilisant les équations de Slutsky, les élasticités-prix compensées de demande ou hicksiennes sont dérivées suivant la formule suivante :

$$
E_{i p}^{c}=E_{i p}^{u}+E_{i} \times w_{h j}
$$

où $E_{i}$ est l'élasticité-dépense.

Sous l'hypothèse de séparabilité des préférences, nous avons procédé à un regroupement des produits afin de faciliter l'analyse empirique et l'interprétation des résultats. Le regroupement des produits est fait de sorte que les produits qui ont tendance à produire le même niveau de satisfaction (substituts proches) soient dans la même catégorie de produits par rapport aux autres produits dont les relations d'interactions sont beaucoup plus générales (Brown et Deaton, 1972). L'idée sous-jacente est que s'il existe une relation entre deux produits appartenant à deux groupes différents, il faudrait que cette relation soit la même pour les autres paires de produits provenant des deux groupes.

\section{Données et problèmes liés aux données}

L'analyse de la demande des produits alimentaires nécessite des données de qualité sur les prix. Les données chronologiques sont, dans une certaine mesure, plus indiquées (Gibson, 2013), mais ces données ne sont pas disponibles sur une période longue dans de nombreux PED comme le Togo. Toutefois, les données d'enquête sur les conditions de vie des ménages qui collectent les informations sur les quantités et les dépenses effectuées par les ménages permettent de calculer les prix unitaires qui exploitées de façon appropriée devraient permettre d'inférer sur le comportement de consommation des ménages (Deaton, 1987). 


\section{Données}

Nous utilisons les données d'enquête sur les conditions de vie des ménages pour estimer les élasticités-prix et revenu de la demande de l'ensemble des produits alimentaires au Togo. Cette enquête est basée sur le questionnaire unifié des indicateurs de base du bien-être (Quibb, 2006). Réalisée au moyen d'un sondage probabiliste ${ }^{1}$, elle rend possible l'extrapolation des résultats de l'échantillon observé à l'ensemble de la population. Elle a consisté en un sondage aréolaire stratifié à deux degrés. La base de sondage des unités primaires est constituée par les 4671 zones de dénombrement (ZD).

$\mathrm{Au}$ premier degré, un échantillon de 300 ZD est tiré avec des probabilités proportionnelles à la taille des $\mathrm{ZD}$. Au deuxième degré, un nombre fixe de 25 ménages a été tiré dans chacune des 300 ZD sélectionnées au premier degré. Au total, l'échantillon de l'enquête est constitué de 7500 ménages dont 2600 en milieu urbain et 4900 en milieu rural. À chaque ménage, le questionnaire Quibb de base qui aborde les questions relatives à la composition du ménage, l'éducation, la santé, l'emploi, les avoirs du ménage et le module Revenu et Dépenses ont été administrés.

\section{Prix unitaires et problèmes liés à l'utilisation des prix unitaires}

En l'absence des prix du marché, une alternative serait d'associer les Indices des prix à la consommation (IPC) aux données d'enquête pour analyser la demande des ménages. Cependant, étant donné que les IPC reposent sur des données collectées seulement sur certains sites ${ }^{2}$, il est difficile

1. Pour des informations détaillées sur la collecte de données de l'enquête QUIBB 2006, voir www.stat-togo.org/nada/index.php.

2. Au Togo, l'Indice harmonisé des prix à la consommation (HIPC) est calculé à partir des données collectées sur 726 points de vente avec comme population de référence, l'ensemble des ménages de l'agglomération de Lomé. que ces prix soient représentatifs des prix des différents produits au niveau national en général et rural en particulier (Deaton et Dupriez, 2011). Nous exploitons donc l'information contenue dans les prix unitaires dans ce papier.

\section{Calcul des prix unitaires}

Pour chaque produit couvert par l'enquête et pour chaque ménage, nous avons les informations sur les quantités et dépenses mensuelles et la fréquence de la consommation annuelle. À partir de ces informations, les dépenses annuelles effectuées par chaque ménage et pour chaque produit sont calculées avant de passer à l'agrégation des produits. Les prix unitaires pour les produits individuels sont donc simplement calculés en faisant le rapport entre les dépenses allouées par le ménage à chaque produit $i$ et leur quantité consommée. La part budgétaire $w_{h i}$ allouée par le ménage $h$ au produit $i$, est donnée par la formule suivante :

$$
w_{h i}=\frac{p_{i h} \times q_{i h}}{x_{h}}
$$

où $p_{i h}$ et $q_{i h}$ sont respectivement le prix unitaire et la quantité du produit $i$ consommé par le ménage $h ; x_{h}$ est la dépense totale de consommation alimentaire du ménage. En notant $D_{h i}$, la part des dépenses allouées à la consommation du produit $i$, le prix unitaire du produit $i$ est déduit comme suit :

$$
p_{i h}=\frac{w_{h i} \times x_{h}}{q_{i h}}=\frac{D_{h i}}{q_{i h}}
$$

Généralement, le calcul des prix unitaires à partir de l'équation (12) engendre des valeurs manquantes liées à des quantités et parts budgétaires nulles pour les produits alimentaires non consommés par les ménages. Ces valeurs manquantes sont traitées suivant la procédure de Deaton et Zaidi (2002). Pour le calcul des prix unitaires des différents produits alimentaires, nous avons utilisé un indice de prix qui prend en compte le poids de chaque 
produit dans son groupe comme suit (voir Deaton, 1997) :

$$
p_{h g}=\prod_{i=1}^{k} p_{h i}{ }^{{ }_{h i}}
$$

où $p_{h g}$ est l'indice géométrique de prix du ménage $h$ pour le groupe de produits $g$ et $k$, le nombre total de produits appartenant au groupe $g$.

\section{Prix unitaires et erreurs de mesure}

Parmi les problèmes posés par l'utilisation des prix unitaires comme proxy des prix du marché, celui des erreurs de mesure semble être le plus sérieux (Capeau et Dercon, 1998 ; Deaton, 1997 ; McKelvey, 2011 ; Tafere et al., 2010). Si les quantités et les dépenses sont rapportées par les ménages avec des erreurs, il est évident que les prix unitaires seront affectés par ces erreurs. Le problème posé par les erreurs de mesure dans les prix unitaires est que ces derniers peuvent être négativement corrélés aux quantités ou positivement corrélés au revenu (dépenses ou parts budgétaires) de façon fallacieuse et donc conduire à des biais dans les estimations des paramètres de la demande. Pour ce faire, l'enquête sur les conditions de vie des ménages au Togo étant menée sur les ménages sélectionnés dans les ZD (clusters) au même moment, nous pouvons faire l'hypothèse qu'il n'y a pas de véritable variation de prix entre ménages appartenant à un même cluster puisque ces derniers réalisent leurs achats de produits alimentaires sur les mêmes marchés (Deaton, 1988).

Dans cette perspective, le problème des erreurs de mesure peut être en partie résolu en considérant les prix unitaires médians des groupes de produits par cluster comme des proxies des prix du marché (Capéau et Dercon, 1998). C'est cette procédure qui est envisagée dans ce papier. En procédant ainsi, nous réduisons considérablement l'effet des valeurs extrêmes et, par conséquent, les erreurs de mesure contenues dans les quantités et dépenses rapportées par chaque ménage. Ainsi, les prix unitaires par cluster vont davantage refléter la variation spatiale des prix entre marchés situés dans différentes zones de dénombrement que l'hétérogénéité des prix entre ménages.

Étant donné que nos données contiennent 300 clusters dans lesquels les ménages ont été tirés, l'analyse statistique de la variation spatiale des prix unitaires est faite sur une base régionale. Les données du tableau 1 montrent que dans l'ensemble il n'y a pas une très grande variation spatiale des prix unitaires des produits entre les régions considérées. Ceci est une indication que les erreurs de mesure auraient été minimisées avec le calcul des prix unitaires médians par cluster même si nous manquons de contrefactuel.

\section{Prix unitaires et effet}

\section{de substitution qualité}

La tendance des ménages plus aisés à consommer les produits de qualité supérieure est susceptible de créer une corrélation positive artificielle entre le revenu et les prix unitaires (ce qui n'est pas le cas avec les prix du marché). Le problème avec le choix de la qualité des produits, c'est que sa présence est susceptible d'engendrer un risque de biais de simultanéité lorsque les prix unitaires sont utilisés comme proxies des prix du marché dans l'estimation des systèmes de demande (Deaton, 1988, 1990). En particulier, si l'effet qualité se révèle pertinent, cela va entraîner une surestimation des élasticitésprix de la demande.

Pour prendre en compte l'effet de substitution qualité dans l'estimation des élasticités-prix de la demande, la part du choix de la qualité dans l'explication des prix unitaires peut être déterminée en régressant le logarithme des prix unitaires sur les dépenses totales et sur les caractéristiques sociodémographiques des ménages (Deaton, 1997), ceci afin de vérifier s'il est nécessaire de corriger les prix unitaires de 
RECHERCHE

Tomgouani LANIE

Tableau 1. Logarithme des prix unitaires moyens à Lomé et dans les cinq régions du Togo

\begin{tabular}{|c|c|c|c|c|c|c|}
\hline \multirow[t]{2}{*}{ Produits et groupes de produits } & \multicolumn{6}{|c|}{ Régions } \\
\hline & Lomé & Maritimes & Plateaux & Centrale & Kara & Savanes \\
\hline Maïs & $\begin{array}{c}5.517 \\
(0.000)\end{array}$ & $\begin{array}{c}6.183 \\
(0.056)\end{array}$ & $\begin{array}{c}6.059 \\
(0.012)\end{array}$ & $\begin{array}{c}6.045 \\
(0.023)\end{array}$ & $\begin{array}{c}6.165 \\
(0.026)\end{array}$ & $\begin{array}{c}6.697 \\
(0.081)\end{array}$ \\
\hline Riz & $\begin{array}{c}5.497 \\
(0.093)\end{array}$ & $\begin{array}{c}5.201 \\
(0.063)\end{array}$ & $\begin{array}{c}5.514 \\
(0.116)\end{array}$ & $\begin{array}{c}5.458 \\
(0.063)\end{array}$ & $\begin{array}{c}5.616 \\
(0.075)\end{array}$ & $\begin{array}{c}5.772 \\
(0.147)\end{array}$ \\
\hline Sorgho & $\begin{array}{c}5.876 \\
(0.000)\end{array}$ & $\begin{array}{c}6.452 \\
(0.000)\end{array}$ & $\begin{array}{c}6.008 \\
(0.186)\end{array}$ & $\begin{array}{c}5.976 \\
(0.075)\end{array}$ & $\begin{array}{c}6.249 \\
(0.104)\end{array}$ & $\begin{array}{l}6.731 \\
(0.124)\end{array}$ \\
\hline Mil & $\begin{array}{c}6.002 \\
(0.000)\end{array}$ & $\begin{array}{c}6.134 \\
(0.000)\end{array}$ & $\begin{array}{c}6.101 \\
(0.018)\end{array}$ & $\begin{array}{c}5.979 \\
(0.160)\end{array}$ & $\begin{array}{c}6.148 \\
(0.064)\end{array}$ & $\begin{array}{c}6.869 \\
(0.118)\end{array}$ \\
\hline Fonio & $\begin{array}{c}5.888 \\
(0.094)\end{array}$ & $\begin{array}{c}5.284 \\
(0.000)\end{array}$ & $\begin{array}{c}5.137 \\
(0.009)\end{array}$ & $\begin{array}{c}5.069 \\
(0.000)\end{array}$ & $\begin{array}{c}5.904 \\
(0.026)\end{array}$ & $\begin{array}{c}5.874 \\
(0.000)\end{array}$ \\
\hline Céréales transformées & $\begin{array}{c}4.801 \\
(0.136)\end{array}$ & $\begin{array}{c}4.268 \\
(0.046)\end{array}$ & $\begin{array}{c}4.791 \\
(0.092)\end{array}$ & $\begin{array}{c}4.639 \\
(0.065)\end{array}$ & $\begin{array}{c}4.857 \\
(0.072)\end{array}$ & $\begin{array}{c}5.148 \\
(0.127)\end{array}$ \\
\hline Racines et tubercules & $\begin{array}{c}5.239 \\
(0.098)\end{array}$ & $\begin{array}{c}5.166 \\
(0.167)\end{array}$ & $\begin{array}{c}5.704 \\
(0.186)\end{array}$ & $\begin{array}{c}5.651 \\
(0.178)\end{array}$ & $\begin{array}{l}6.010 \\
(0.442)\end{array}$ & $\begin{array}{l}5.437 \\
(0.321)\end{array}$ \\
\hline Légumineuses et oléagineux & $\begin{array}{c}4.666 \\
(0.076)\end{array}$ & $\begin{array}{c}5.073 \\
(0.116)\end{array}$ & $\begin{array}{c}5.159 \\
(0.154)\end{array}$ & $\begin{array}{c}4.835 \\
(0.103)\end{array}$ & $\begin{array}{c}5.151 \\
(0.218)\end{array}$ & $\begin{array}{c}5.143 \\
(0.223)\end{array}$ \\
\hline Huiles et matières grasses & $\begin{array}{c}4.532 \\
(0.037)\end{array}$ & $\begin{array}{c}4.313 \\
(0.034)\end{array}$ & $\begin{array}{c}4.517 \\
(0.049)\end{array}$ & $\begin{array}{c}4.138 \\
(0.023)\end{array}$ & $\begin{array}{c}4.446 \\
(0.077)\end{array}$ & $\begin{array}{c}4.564 \\
(0.074)\end{array}$ \\
\hline Fruits & $\begin{array}{c}4.613 \\
(0.108)\end{array}$ & $\begin{array}{c}4.630 \\
(0.298)\end{array}$ & $\begin{array}{c}4.819 \\
(0.084)\end{array}$ & $\begin{array}{c}4.927 \\
(0.084)\end{array}$ & $\begin{array}{c}5.426 \\
(0.110)\end{array}$ & $\begin{array}{c}4.926 \\
(0.060)\end{array}$ \\
\hline Légumes & $\begin{array}{c}4.388 \\
(0.060)\end{array}$ & $\begin{array}{c}4.147 \\
(0.060)\end{array}$ & $\begin{array}{c}4.249 \\
(0.116)\end{array}$ & $\begin{array}{c}3.904 \\
(0.048)\end{array}$ & $\begin{array}{c}4.019 \\
(0.081)\end{array}$ & $\begin{array}{c}4.115 \\
(0.113)\end{array}$ \\
\hline Viandes & $\begin{array}{c}5.879 \\
(0.218)\end{array}$ & $\begin{array}{c}6.211 \\
(0.360)\end{array}$ & $\begin{array}{c}5.945 \\
(0.326)\end{array}$ & $\begin{array}{c}5.681 \\
(0.173)\end{array}$ & $\begin{array}{c}4.887 \\
(0.308)\end{array}$ & $\begin{array}{c}5.469 \\
(0.199)\end{array}$ \\
\hline Poissons/Crustacés & $\begin{array}{c}5.562 \\
(0.091)\end{array}$ & $\begin{array}{c}5.318 \\
(0.027)\end{array}$ & $\begin{array}{c}5.282 \\
(0.020)\end{array}$ & $\begin{array}{c}4.680 \\
(0.088)\end{array}$ & $\begin{array}{c}4.808 \\
(0.071)\end{array}$ & $\begin{array}{c}4.666 \\
(0.165)\end{array}$ \\
\hline Fufs & $\begin{array}{c}5.118 \\
(0.251)\end{array}$ & $\begin{array}{c}4.252 \\
(0.140)\end{array}$ & $\begin{array}{c}4.222 \\
(0.154)\end{array}$ & $\begin{array}{c}4.198 \\
(0.169)\end{array}$ & $\begin{array}{c}4.518 \\
(0.129)\end{array}$ & $\begin{array}{c}4.647 \\
(0.083)\end{array}$ \\
\hline Lait et produits assimilés & $\begin{array}{c}5.183 \\
(0.061)\end{array}$ & $\begin{array}{c}4.703 \\
(0.069)\end{array}$ & $\begin{array}{c}4.663 \\
(0.060)\end{array}$ & $\begin{array}{c}4.976 \\
(0.056)\end{array}$ & $\begin{array}{c}5.068 \\
(0.056)\end{array}$ & $\begin{array}{c}4.898 \\
(0.084)\end{array}$ \\
\hline Sucre, boissons et excitants & $\begin{array}{c}4.846 \\
(0.199)\end{array}$ & $\begin{array}{c}4.458 \\
(0.166)\end{array}$ & $\begin{array}{c}4.678 \\
(0.227)\end{array}$ & $\begin{array}{c}4.793 \\
(0.397)\end{array}$ & $\begin{array}{c}5.059 \\
(0.258)\end{array}$ & $\begin{array}{l}5.070 \\
(0.164)\end{array}$ \\
\hline Conserves et condiments & $\begin{array}{c}4.173 \\
(0.092)\end{array}$ & $\begin{array}{c}3.606 \\
(0.061)\end{array}$ & $\begin{array}{c}3.968 \\
(0.094)\end{array}$ & $\begin{array}{c}3.608 \\
(0.052)\end{array}$ & $\begin{array}{c}3.701 \\
(0.102)\end{array}$ & $\begin{array}{c}3.762 \\
(0.090)\end{array}$ \\
\hline Aliments hors ménage & $\begin{array}{c}6.803 \\
(0.128)\end{array}$ & $\begin{array}{c}6.114 \\
(0.121)\end{array}$ & $\begin{array}{c}6.154 \\
(0.122)\end{array}$ & $\begin{array}{c}6.288 \\
(0.194)\end{array}$ & $\begin{array}{c}5.851 \\
(0.150)\end{array}$ & $\begin{array}{c}5.553 \\
(0.102)\end{array}$ \\
\hline Autres produits alimentaires & $\begin{array}{c}5.536 \\
(0.026)\end{array}$ & $\begin{array}{c}5.515 \\
(0.030)\end{array}$ & $\begin{array}{c}5.909 \\
(0.052)\end{array}$ & $\begin{array}{c}5.649 \\
(0.041)\end{array}$ & $\begin{array}{c}6.070 \\
(0.009)\end{array}$ & $\begin{array}{c}5.264 \\
(0.052)\end{array}$ \\
\hline Produits non alimentaires & $\begin{array}{c}7.024 \\
(0.142)\end{array}$ & $\begin{array}{c}6.118 \\
(0.251)\end{array}$ & $\begin{array}{c}6.536 \\
(0.287)\end{array}$ & $\begin{array}{c}6.401 \\
(0.114)\end{array}$ & $\begin{array}{c}6.321 \\
(0.232)\end{array}$ & $\begin{array}{c}6.269 \\
(0.178)\end{array}$ \\
\hline
\end{tabular}

Source : calcul de l'auteur d'après les données d'enquête sur les conditions de vie des ménages au Togo (QUIBB 2006). 
l'effet qualité avant l'estimation des élasticités-prix et revenu de la demande suivant l'équation suivante :

$\ln p_{h i c}=\alpha+\eta \ln x_{h c}+\vartheta \cdot Z_{h c}+\chi \ln P_{i c}+u_{h i c}$

où $\ln p_{h i c}$ représente le logarithme du prix unitaire du produit alimentaire $i$ consommé par le ménage $h$ appartenant au cluster $c ; \ln x_{h c}$ est le logarithme des dépenses totales du ménage allouées à la consommation des produits alimentaires; $Z_{h c}$ est un vecteur des caractéristiques sociodémographiques du ménage. Le vecteur $Z_{h c}$ renferme la taille du ménage, la situation d'emploi, le sexe, l'âge et l'âge au carré, le niveau d'éducation du chef du ménage et la composition du ménage (structure par âge des membres du ménage) formée des variables muettes représentant les différents niveaux d'âges des membres du ménage (0 à 4 ans ; 5 à 9 ans ; 10 à 14 ans ; 15 à 54 ans ; et 55 ans et plus). La variable ln $P_{i c}$ représente le logarithme des prix inobservés des produits dans le cluster $c$ (prix du marché) ; $\alpha, \eta, \vartheta$ et $\chi$ sont les paramètres à estimer et $u_{\text {hic }}$ est le terme d'erreurs. L'équation (14) telle que décrite n'est pas identifiable, la variable $P_{i c}$ étant inobservable. Donc pour annihiler l'effet des prix et pour pouvoir estimer l'équation (14), toutes les variables du modèle sont extraites de leurs valeurs moyennes par cluster (Deaton, 1987) suivant l'équation suivante :

$$
\begin{aligned}
& \left(\ln p_{h i c}-\ln \bar{p}_{i c}\right)=\beta\left(\ln x_{h c}-\ln \bar{x}_{c}\right) \\
& +\gamma\left(Z_{h c}-\bar{Z}_{c}\right)+\left(u_{h i c}-\bar{u}_{i c}\right)
\end{aligned}
$$

L'équation (15) est estimée par la méthode des moindres carrés ordinaires par cluster (voir Deaton, 1987) et seul le paramètre d'intérêt $\beta$ qui représente l'effet de substitution qualité est reporté dans le tableau 2. Dans ce tableau, les pauvres et les non-pauvres sont définis à partir du seuil de pauvreté au niveau national qui est de 242094 FCFA en 2006. Selon les résultats des estimations, au niveau national, les élasticités du choix de la qualité des produits se sont révélées significatives seulement pour sept (7) produits sur les vingt (20) produits considérés. Il s'agit du riz, des céréales transformées, des racines et tubercules, du sucre, boissons et excitants, des conserves et condiments, des aliments hors ménage et des produits non alimentaires. Ces élasticités varient selon le milieu de résidence des ménages et leur statut de pauvreté.

Toutefois, il n'y a aucune tendance qui se dégage par rapport à ces deux catégories exceptés les produits riz et céréales transformées où ces élasticités se sont révélées significatives en milieu urbain et pour les ménages non pauvres.

Suivant le milieu de résidence des ménages, ces élasticités se sont révélées de plus grande ampleur en milieu rural qu'en milieu urbain pour les produits alimentaires racines et tubercules, conserves et condiments, aliments hors ménage et inversement pour les produits sucre, boissons et excitants et produits non alimentaires. Suivant le statut de pauvreté des ménages, ces élasticités sont de plus grande ampleur pour la catégorie nonpauvres par rapport à la catégorie pauvres pour les produits sucre, boissons et excitants, conserves, condiments et produits non alimentaires.

Dans l'ensemble, les résultats du tableau 2 nous amènent à supposer que les ménages plus aisés ont tendance à consommer les produits de meilleure qualité que les ménages pauvres.

Mais ce qui est important dans notre analyse de l'effet qualité des produits, c'est l'ampleur de ces élasticités qui nous permet de juger de la nécessité de corriger ou non les prix unitaires de l'effet de substitution par la qualité avant l'estimation des élasticités-prix de la demande. En considérant les résultats du tableau 2, dans l'ensemble, les élasticités du choix de la qualité sont comprises entre -0.461 pour 
RECHERCHE

Tomgouani LANIE

Tableau 2. Résultats des estimations des élasticités du choix de la qualité des produits

\begin{tabular}{|c|c|c|c|c|c|}
\hline \multirow[t]{2}{*}{ Produits et groupes de produits } & \multicolumn{5}{|c|}{ Élasticités du choix de la qualité des produits } \\
\hline & National & Urbain & Rural & Pauvres & Non-pauvres \\
\hline \multirow[t]{2}{*}{ Maïs } & 0.001 & -0.010 & 0.002 & 0.004 & $0.021 * * *$ \\
\hline & $(0.004)$ & $(0.009)$ & $(0.004)$ & $(0.005)$ & $(0.008)$ \\
\hline \multirow[t]{2}{*}{ Riz } & $0.038 * * *$ & $0.137 * * *$ & $-0.014 * * *$ & -0.007 & $0.096 * * *$ \\
\hline & $(0.005)$ & $(0.012)$ & $(0.005)$ & $(0.006)$ & $(0.009)$ \\
\hline \multirow[t]{2}{*}{ Sorgho } & $-0.006 * * *$ & $-0.010 * * *$ & -0.003 & $-0.015 * * *$ & $-0.011 * * *$ \\
\hline & $(0.002)$ & $(0.003)$ & $(0.003)$ & $(0.003)$ & $(0.003)$ \\
\hline \multirow[t]{2}{*}{ Mil } & $-0.007 * * *$ & $0.008^{* *}$ & $-0.013 * * *$ & $-0.018 * * *$ & $-0.009 * * *$ \\
\hline & $(0.002)$ & $(0.003)$ & $(0.003)$ & $(0.004)$ & $(0.003)$ \\
\hline \multirow[t]{2}{*}{ Fonio } & $-0.002 * * *$ & $-0.003 * * *$ & $-0.002 * * *$ & $-0.002 * * *$ & $-0.002 * *$ \\
\hline & $(0.0004)$ & $(0.001)$ & $(0.001)$ & $(0.0004)$ & $(0.001)$ \\
\hline \multirow{2}{*}{ Céréales transformées } & $0.012 * * *$ & $0.111 * * *$ & $-0.034 * * *$ & $-0.012 * * *$ & $0.074 * * *$ \\
\hline & $(0.004)$ & $(0.009)$ & $(0.003)$ & $(0.004)$ & $(0.008)$ \\
\hline \multirow[t]{2}{*}{ Racines et tubercules } & $0.129 * * *$ & $0.059 * * *$ & $0.159 * * *$ & $0.149 * * *$ & $0.057 * * *$ \\
\hline & $(0.008)$ & $(0.012)$ & $(0.011)$ & $(0.011)$ & $(0.013)$ \\
\hline \multirow[t]{2}{*}{ Légumineuses et oléagineux } & $-0.022 * * *$ & $-0.096 * * *$ & $0.013^{*}$ & -0.002 & $-0.075 * * *$ \\
\hline & $(0.006)$ & $(0.008)$ & $(0.007)$ & $(0.008)$ & $(0.009)$ \\
\hline \multirow[t]{2}{*}{ Huiles et matières grasses } & 0.0001 & $-0.009 * *$ & 0.005 & -0.004 & -0.0001 \\
\hline & $(0.003)$ & $(0.004)$ & $(0.004)$ & $(0.004)$ & $(0.004)$ \\
\hline \multirow[t]{2}{*}{ Fruits } & $-0.069 * * *$ & $-0.081 * * *$ & $-0.059 * * *$ & $-0.050 * * *$ & $-0.110 * * *$ \\
\hline & $(0.005)$ & $(0.008)$ & $(0.007)$ & $(0.007)$ & $(0.009)$ \\
\hline \multirow[t]{2}{*}{ Légumes } & $-0.043 * * *$ & $-0.071 * * *$ & $-0.029 * * *$ & $-0.059 * * *$ & $-0.039 * * *$ \\
\hline & $(0.004)$ & $(0.005)$ & $(0.005)$ & $(0.005)$ & $(0.005)$ \\
\hline \multirow[t]{2}{*}{ Viandes } & $-0.182 * * *$ & $-0.179 * * *$ & $-0.179 * * *$ & $-0.191 * * *$ & $-0.222 * * *$ \\
\hline & $(0.009)$ & $(0.015)$ & $(0.011)$ & $(0.012)$ & $(0.015)$ \\
\hline \multirow[t]{2}{*}{ Poissons/Crustacés } & $-0.021 * * *$ & 0.005 & $-0.033 * * *$ & $-0.038 * * *$ & -0.003 \\
\hline & $(0.004)$ & $(0.007)$ & $(0.005)$ & $(0.005)$ & $(0.007)$ \\
\hline \multirow[t]{2}{*}{ Eufs } & $-0.029 * * *$ & $-0.100 * * *$ & -0.001 & $-0.015 * * *$ & $-0.016 * *$ \\
\hline & $(0.003)$ & $(0.008)$ & $(0.002)$ & $(0.003)$ & $(0.008)$ \\
\hline \multirow[t]{2}{*}{ Lait et produits assimilés } & $-0.033 * * *$ & $-0.064 * * *$ & $-0.021 * * *$ & $-0.021 * * *$ & $-0.053 * * *$ \\
\hline & $(0.003)$ & $(0.005)$ & $(0.004)$ & $(0.004)$ & $(0.006)$ \\
\hline \multirow[t]{2}{*}{ Sucre, boissons et excitants } & $0.044 * * *$ & $0.089 * * *$ & $0.022 * * *$ & -0.008 & $0.151 * * *$ \\
\hline & $(0.007)$ & $(0.012)$ & $(0.008)$ & $(0.009)$ & $(0.013)$ \\
\hline \multirow[t]{2}{*}{ Conserves et condiments } & $0.129 * * *$ & $0.017 * * *$ & $0.112^{* * *}$ & $0.109 * * *$ & $0.144 * * *$ \\
\hline & $(0.004)$ & $(0.007)$ & $(0.005)$ & $(0.005)$ & $(0.007)$ \\
\hline \multirow[t]{2}{*}{ Aliments hors ménage } & $0.055 * * *$ & $0.042 * * *$ & $0.059 * * *$ & $0.066 * * *$ & $0.049 * * *$ \\
\hline & $(0.003)$ & $(0.007)$ & $(0.004)$ & $(0.004)$ & $(0.007)$ \\
\hline \multirow[t]{2}{*}{ Autres produits alimentaires } & $-0.461 * * *$ & $-0.852 * * *$ & $-0.288 * * *$ & $-0.163 * * *$ & $-0.264 * * *$ \\
\hline & $(0.028)$ & $(0.055)$ & $(0.032)$ & $(0.029)$ & $(0.061)$ \\
\hline \multirow[t]{2}{*}{ Produits non alimentaires } & $1.022 * * *$ & $1.166 * * *$ & $0.958 * * *$ & $1.002 * * *$ & $1.226 * * *$ \\
\hline & $(0.015)$ & $(0.028)$ & $(0.017)$ & $(0.021)$ & $(0.022)$ \\
\hline Obs. & 7500 & 2600 & 4900 & 3885 & 3615 \\
\hline
\end{tabular}

Note : les écarts-types robustes sont entre parenthèses et $\left({ }^{* * *}\right),\left({ }^{* *}\right)$ et $\left({ }^{*}\right)$ désignent les niveaux de significativité de $1 \%, 5 \%$ et $10 \%$ respectivement. 
les autres produits alimentaires et 0.129 pour les racines et tubercules, conserves et condiments. Nous estimons donc que ces élasticités du choix de la qualité des produits par les ménages sont assez faibles pour nécessiter la correction pour deux raisons principales.

La première raison est que Deaton (1988, 1990, 1997) a montré au niveau théorique la pertinence de la prise en compte de l'effet de substitution par la qualité dans l'estimation des élasticitésprix de la demande mais n'a pas défini un seuil à partir duquel il devient nécessaire de corriger les élasticités-prix de l'effet qualité. La seconde est que Tafere et al. (2010), par exemple, ont trouvé des élasticités du choix de la qualité des produits variant entre -0.018 pour le Sorgho et 0.1722 pour le groupe de produits sucre et sel en Éthiopie. Ils en ont conclu que ces élasticités du choix de la qualité n'étaient pas aussi importantes pour que le problème du choix de la qualité soit corrigé avant l'estimation des élasticités-prix de la demande. Nous exploitons donc, dans ce papier, l'information contenue dans les prix unitaires par cluster pour estimer les élasticités de demande des produits alimentaires au Togo.

\section{Problèmes économétriques et stratégie d'estimation}

L'estimation des systèmes de demande avec les données d'enquête ménages pose deux problèmes économétriques majeurs. Le premier problème est le problème de sélection lié à la présence de dépenses ou de parts budgétaires nulles dans la structure de dépenses des ménages. Le second problème est celui de l'endogénéité du logarithme des dépenses totales de consommation par rapport aux parts budgétaires, le revenu étant approximé par les dépenses totales. Pour résoudre les problèmes d'endogénéité et de sélection, nous adoptons respectivement les approches en deux étapes développées par Blundell et Robin (1999) et Shonkwiler et Yen (1999).

\section{Problème de sélection}

L'une des hypothèses sur lesquelles repose l'analyse empirique de la demande postule que tous les biens considérés sont essentiels, c'est-à-dire qu'en toute circonstance, ces biens sont achetés ou consommés par les ménages (Deaton, 1986). Cependant, il est fréquemment observé dans les données d'enquête sur la consommation des ménages, des parts budgétaires nulles dans la structure de dépenses des ménages. Le regroupement des produits devrait permettre d'éliminer la présence de parts budgétaires nulles, mais le tableau 3 montre que la présence des parts budgétaires nulles est prépondérante dans nos données. La préoccupation est qu'elles sont susceptibles d'engendrer le problème de sélection.

Plusieurs causes sont identifiées comme étant à l'origine de l'observation des parts budgétaires nulles dans la structure de dépenses des ménages : (i) les erreurs lorsque les ménages rapportent leurs dépenses de consommation pendant le déroulement de l'enquête; (ii) l'absence de consommation d'un produit durant la période d'enquête (en particulier lorsque la période de consommation couverte par l'enquête est relativement courte); (iii) la présence des produits non consommés de façon permanente ; (iv) et la présence de la nonconsommation optimale (solution en coin).

Toutes les causes énumérées n'étant pas susceptibles de poser des problèmes statistiques, nous analysons donc ces causes à la lumière de nos données afin de corriger de façon efficace les problèmes statistiques que les parts budgétaires nulles sont susceptibles d'engendrer.

\section{Origine des parts budgétaires nulles et problème de sélection}

L'enjeu associé à l'origine des parts budgétaires nulles est que si la présence des parts 
RECHERCHE

Tomgouani LANIE

Tableau 3. Pourcentages des parts budgétaires nulles dans la structure de dépenses des ménages

\begin{tabular}{lccccc}
\hline Produits et groupes de produits & \multicolumn{5}{c}{ Parts budgétaires (\%) } \\
\cline { 2 - 6 } & National & Urbain & Rural & Pauvres & Non-pauvres \\
\hline Maïs & 09.936 & 12.543 & 08.553 & 09.735 & 10.152 \\
Riz & 12.977 & 10.812 & 14.125 & 14.808 & 11.009 \\
Sorgho & 73.620 & 92.497 & 63.605 & 62.838 & 85.201 \\
Mil & 83.769 & 94.575 & 78.036 & 75.406 & 92.752 \\
Fonio & 98.173 & 99.154 & 97.653 & 97.837 & 98.534 \\
Céréales transformées & 13.310 & 08.619 & 15.799 & 17.409 & 08.907 \\
Racines et tubercules & 11.416 & 05.425 & 14.595 & 17.280 & 05.118 \\
Légumineuses et oléagineux & 11.176 & 11.697 & 10.900 & 11.847 & 10.456 \\
Huiles et matières grasses & 10.216 & 06.426 & 12.227 & 13.314 & 06.888 \\
Fruits & 53.054 & 29.935 & 65.319 & 67.062 & 38.008 \\
Légumes & 03.214 & 03.463 & 03.082 & 03.425 & 02.988 \\
Viandes & 28.941 & 26.241 & 30.374 & 31.419 & 26.279 \\
Poissons/Crustacés & 15.778 & 10.466 & 18.596 & 20.783 & 10.401 \\
CEufs & 76.034 & 55.983 & 86.671 & 88.257 & 62.905 \\
Lait et produits assimilés & 70.152 & 52.443 & 79.547 & 82.694 & 56.680 \\
Sucre, boissons et excitants & 12.750 & 10.504 & 13.942 & 16.122 & 09.129 \\
Conserves et condiments & 01.707 & 02.886 & 01.082 & 01.004 & 02.462 \\
Aliments hors ménage & 50.667 & 30.012 & 61.525 & 64.666 & 35.629 \\
Autres produits alimentaires & 87.489 & 79.838 & 91.549 & 93.278 & 81.272 \\
Produits non alimentaires & 00.067 & 00.077 & 00.061 & 00.129 & 00.000 \\
\hline
\end{tabular}

Source : calcul de l'auteur d'après les données d'enquête sur les conditions de vie des ménages au Togo (QUIBB 2006).

budgétaires nulles est due aux erreurs exogènes des ménages lorsqu'ils rapportent leurs dépenses ou à l'existence de la nonconsommation permanente, la solution évidente serait de supprimer les observations pour lesquelles les parts budgétaires sont nulles. Il n'y aurait donc aucun biais dans les estimations puisque ne provenant pas d'un comportement de consommation optimisée des ménages. Cependant, si la présence des parts budgétaires nulles provient du comportement de consommation optimale des ménages, supprimer ces observations conduirait à des biais dans les estimations. Par rapport à nos données sur les dépenses des ménages, l'enquête étant menée sur une base annuelle, la seconde cause n'est pas envisageable dans nos données.

Mais pour les trois autres causes restantes, mise à part l'occurrence des erreurs exogènes des ménages en rapportant leurs dépenses, les parts budgétaires nulles ne peuvent provenir que de la présence de deux catégories de consommateurs. La première catégorie de consommateurs concerne ceux qui ne consommeront jamais un produit pour des raisons non économiques comme leur croyance religieuse, leur culture ou pour des raisons de santé (non-consommation permanente). La seconde catégorie de consommateurs 
concerne ceux qui ne consomment pas un produit du fait de l'existence d'un substitut parfait ou ceux pour lesquels la non-consommation d'un produit résulte d'un processus d'optimisation. Cette dernière catégorie de ménages constitue des consommateurs potentiels des produits pour lesquels nous avons observé des dépenses nulles dans leur structure de dépenses. En effet, si les prix relatifs des biens changeaient ou si leur revenu augmentait, ces derniers pourraient consommer les produits qu'ils ne consommeraient pas et vice-versa. Malgré l'importance des parts budgétaires nulles dans la structure de dépense des ménages dans nos données, nous n'avons malheureusement aucune information sur la cause fondamentale de ces parts budgétaires nulles. Pour éviter tout éventuel biais de sélection, nous faisons l'hypothèse forte qu'une grande partie de ces parts budgétaires nulles est liée aux décisions économiques des ménages.

\section{Correction du problème de sélection}

Suivant la procédure de Shonkwiler et Yen (1999), la correction du problème de sélection est faite à travers l'estimation du système d'équations suivant :

$w_{h i}^{*}=f\left(x_{h i}, \mu_{i}\right)+\varepsilon_{h i} ; d_{h i}^{*}=z_{h i} \theta_{i}+v_{h i}$

$d_{h i}=\left\{\begin{array}{l}1 \text { si } d_{h i}^{*}>0 \\ 0 \text { si } d_{h i}^{*} \leq 0\end{array}\right.$ avec $w_{h i}=d_{h i} w_{h i}^{*}$

où $h$ et $i$ sont respectivement des indices pour les ménages et les produits ou groupe de produits ; $w_{h i}$ et $d_{h i}$ sont respectivement les parts budgétaires observées et une variable dichotomique indiquant si le ménage est consommateur ou non du $i^{\grave{e}}$ produit ou groupe de produits; $w_{h i}^{*}$ et $d_{h i}^{*}$ sont les variables latentes correspondantes ; $x_{h i}$ et $z_{h i}$ sont des vecteurs de variables explicatives ; $\mu_{i}$ et $\theta_{i}$ sont des vecteurs de paramètres : et $u_{h i}$ et $v_{h i}$ sont les termes d'erreurs aléatoires. Le système d'équations (16) implique que chaque $i^{\grave{e}}$ équation $w_{h i}$ est observée seulement pour des valeurs non négatives. Ainsi, si une grande fraction des parts budgétaires $w_{i}$ est nulle, alors les représenter par une distribution continue serait inapproprié. Pour chaque produit $i$, les termes d'erreurs $u_{i} \cdot v_{i}^{\prime}$ sont distribués comme des variables normales bivariées avec une covariance $\operatorname{cov}\left(u_{i}, v_{i}\right)=\delta_{i}^{2}$ et une espérance inconditionnelle donnée par:

$$
\begin{gathered}
E\left(w_{h i} \mid x_{h i}, z_{h i}\right)=\Phi\left(z_{h i} \theta_{i}\right) f\left(x_{h i}, \mu_{i}\right) \\
+\delta_{i} \phi\left(z_{h i} \theta_{i}\right)
\end{gathered}
$$

Avec l'équation (18), nous pouvons réécrire l'équation (17) comme suit :

$$
\begin{gathered}
w_{h i}=\Phi\left(z_{h i} \theta_{i}\right) f\left(x_{h i}, \mu_{i}\right) \\
+\delta_{i} \phi\left(z_{h i} \theta_{i}\right)+\varepsilon_{h i}
\end{gathered}
$$

où $\mathcal{E}_{h i}=w_{h i}-E\left(w_{h i} \mid x_{h i}, z_{h i}\right) ; \Phi(\cdot)$ et $\phi(\cdot)$ sont respectivement les fonctions univariées de densité cumulative et de densité de probabilité. À partir des équations (18) et (19), la procédure en deux étapes en utilisant toutes les observations devient possible. La procédure de Shonkwiler et Yen (1999) consiste dans une première étape à obtenir les paramètres estimés $\hat{\theta}_{i}$ de $\theta_{i}$ à travers l'estimation par le maximum de vraisemblance du modèle probit en utilisant les données bivariées de la variable $d_{h i}$ pour chaque produit alimentaire $i$ et dans une deuxième étape à calculer $\Phi\left(z_{h i}, \hat{\theta}_{i}\right)$ et $\phi\left(z_{h i}, \hat{\theta}_{i}\right)$ puis estimer les paramètres $\mu_{i}$ et $\delta_{i}$ du système suivant par le maximum de vraisemblance ou la procédure SUR :

$w_{h i}=\Phi\left(z_{h i}, \hat{\theta}_{i}\right) f\left(x_{h i}, \mu_{i}\right)+\delta_{i} \phi\left(z_{h i}^{\prime} \hat{\theta}_{i}\right)+\xi_{h i}$

où

$$
\begin{aligned}
\xi_{h i} & =\varepsilon_{h i}-\left[\Phi\left(z_{h i}^{\prime} \hat{\theta}_{i}\right)-\Phi\left(z_{h i}^{\prime} \theta_{i}\right)\right] f\left(x_{h i}, \mu_{i}\right) \\
& +\delta_{i}\left[\phi\left(z_{h i} \theta_{i}\right)-\phi\left(z_{h i} \hat{\theta}_{i}\right)\right]
\end{aligned}
$$

Notons qu'avec l'application de l'approche de Shonkwiler et Yen (1999) sur les données censurées, il n'est plus possible 
d'imposer la contrainte d'additivité à travers les restrictions paramétriques. Pour résoudre ce problème, nous avons adopté l'approche de Yen et al. (2003) qui consiste à estimer premièrement $(n-1)$ équations et à considérer la $n^{e}$ équation comme résiduelle. Aussi, les termes d'erreurs de l'équation (20) sont hétéroscédastiques. Pour tenter de corriger ce problème, nous avons utilisé les écarts-types robusts.

\section{Problème d'endogénéité des dépenses totales}

La procédure en deux étapes de Blundell et Robin (1999) consiste à régresser, dans une première étape, le logarithme des dépenses totales des ménages sur un ensemble de variables exogènes incluant celles qui peuvent influencer directement les parts budgétaires. Ensuite, les résidus obtenus de cette première étape sont introduits ensemble avec le logarithme des dépenses totales dans la seconde étape comme variable explicative dans le système d'équations de parts budgétaires. Blundell et Robin (1999) ont souligné que l'estimateur des Moindres carrés ordinaires (MCO) dans la seconde étape est équivalent à l'estimateur des Doubles moindres carrés (DMC) et le test de significativité du coefficient des résidus obtenus dans la régression augmentée de la deuxième étape sert comme un test d'exogénéité du logarithme des dépenses totales des ménages, c'est-à-dire que si le coefficient associé au résidu de la deuxième étape est significatif, alors le logarithme des dépenses totales est exogène.

\section{Stratégie d'estimation du modèle QUAIDS}

Suivant la procédure en deux étapes de Shonkwiler et Yen (1999), la première étape consiste à estimer la probabilité d'achat des ménages pour les différents produits alimentaires suivant l'équation suivante :

$$
\begin{aligned}
d_{h i} & =\pi_{0}+\sum_{j} \pi_{i j} \cdot \ln p_{j}+\pi_{x} \ln x_{h} \\
& +\sum_{k} \pi_{1 k} \cdot Z_{h k}+\sum_{m=1}^{5} \eta_{3 r} \cdot D_{r} \\
& +\sum_{l} \pi_{2 l} \cdot A_{h l}+v_{i}
\end{aligned}
$$

où $d_{h i}=1$ si le ménage $h$ a consommé le $i^{\grave{e}}$ produit alimentaire $\left(w_{h i}>0\right)$ et $d_{h i}=0$ sinon. La variable $Z_{h k}$ représente la taille du ménage et les caractéristiques sociodémographiques du chef de ménage (situation d'emploi, sexe, âge, âge au carré et le niveau d'éducation), $D_{r}$ représente les variables muettes régionales, $A_{h l}$ représente les actifs du ménage (le nombre de pièces, la superficie des terres possédées par le ménage, le nombre de bétails et autres gros animaux possédés par le ménage, le nombre de moutons, chèvres et autres animaux de taille moyenne possédés par le ménage, la possession de l'électricité, d'un réfrigérateur, congélateur, cuisinière/ réchaud) et $v_{i}$, le terme d'erreurs. Le modèle probit (équation 22) est estimé par le maximum de vraisemblance pour chaque produit alimentaire.

Mais avant l'estimation de la seconde étape, le logarithme des dépenses totales est régressé sur un ensemble de variables explicatives suivant l'équation suivante :

$$
\begin{aligned}
\ln x_{h} & =\eta_{0}+\sum_{j} \eta_{i j} \cdot \ln p_{j} \\
& +\sum_{k} \eta_{1 k} \cdot Z_{h k}+\sum_{l} \eta_{2 l} \cdot W_{h l} \\
& +\sum_{r=1}^{5} \eta_{3 r} \cdot D_{r}+e_{h}
\end{aligned}
$$

où les variables $Z_{h k}$ et $D_{r}$ sont les mêmes que celles décrites dans l'équation (22); $W_{h l}$ est un ensemble de variables représentant la richesse du ménage (le nombre de pièces, la superficie des terres possédées par le ménage, le nombre de bétails et autres gros animaux possédés par le ménage, le nombre de moutons, chèvres et autres animaux de taille moyenne possédés 
par le ménage, l'électricité, le matériau du toit de la maison, type d'aisance utilisé par le ménage, voiture à usage non commercial, moto à usage personnel, bicyclettevélo, machine à coudre, fer à repasser charbon, fer à repasser électrique, TV-lecteur, radio, ventilateur, climatiseur, antenne parabolique-décodeur, téléphone mobileportable, téléphone fixe, fauteuils-canapés, armoires-buffets, lit, micro-ordinateur/imprimante, chauffe-eau, suppresseur) et $e_{h}$, un terme d'erreurs normalement distribué. L'équation (23) est estimée par les MCO. Les résidus $\hat{e}_{h}$ sont calculés et comme les paramètres $\Phi\left(z_{h i}^{\prime} \hat{\theta}_{i}\right)$ et $\phi\left(z_{h i}^{\prime} \hat{\theta}_{i}\right)$ des résultats d'estimation de l'équation (22), ils entrent dans la deuxième étape de l'estimation (Tafere et al., 2010). En définitive, le modèle estimé qui prend en compte à la fois le problème de sélection et d'endogénéité des dépenses est exprimé comme suit :

$$
\begin{aligned}
& w_{h i}=\Phi\left(z_{h i}^{\prime} \hat{\theta}_{i}\right)\left\{\alpha_{i}+\sum_{j=1}^{n} \gamma_{i j} p_{j}\right. \\
& \left.+\beta_{i} \ln \left[\frac{x}{a(p)}\right]+\frac{\lambda_{i}}{b(p)}\left\{\ln \left[\frac{x}{a(p)}\right]\right\}^{2}+\omega_{i} \hat{e}_{h}\right\} \\
& +\delta_{i} \phi\left(z_{h i}^{\prime} \hat{\theta}_{i}\right)+\xi_{h i}
\end{aligned}
$$

Les paramètres du modèle sont estimés suivant la procédure d'estimation de Poi (2008) dont la programmation a été modifiée pour prendre en compte les problèmes d'endogénéité et de sélection. Dans cette procédure d'estimation, les contraintes d'additivité, d'homogénéité et de symétrie soulignées plus haut sont imposées et le modèle est estimé comme un système NLSSURE (Non-linear System of Seemingly Unrelated Regression Equations). La valeur du paramètre $\alpha_{0}$ de l'équation (2) est choisie comme étant la valeur juste en dessous de la valeur minimale du logarithme des dépenses $\ln x$ (Deaton et Muellbauer, 1980 ; Poi, 2008, 2012). Pour imposer la contrainte d'additivité, l'équation (20) du modèle QUAIDS représentant le groupe de produits non alimentaires a été écartée et les paramètres du modèle sont estimés sur les 19 premières équations par la méthode IFGNLS (Iterated Feasible Generalized Non-Linear Least Squared). Les paramètres du modèle QUAIDS pour la $20^{\circ}$ équation et les élasticités de l'équation (20) du modèle sont obtenus en exploitant les relations de restrictions d'homogénéité et de symétrie.

\section{Résultats des estimations}

Les estimations du modèle QUAIDS sont performantes. Les résultats montrent la pertinence de la prise en compte du problème de sélection lié à la présence des parts budgétaires nulles dans la structure de dépenses des ménages. Quant au problème d'endogénéité, les résultats montrent l'exogénéité des dépenses totales des ménages aux parts budgétaires ${ }^{3}$.

Les résultats des estimations des élasticités-prix compensées de la demande calculés au point moyen dans le tableau 4 montrent que, dans l'ensemble, ces élasticités ont des signes conformes à la théorie économique, c'est-à-dire des signes négatifs indiquant que lorsque le prix d'un bien augmente, toute chose égale par ailleurs, la demande pour ce bien diminue. Au niveau national, les résultats montrent que les céréales comme le maïs, le riz et les céréales transformées sont caractérisées par une demande inélastique alors que les céréales comme le sorgho, le mil et le fonio sont caractérisées par une demande très élastique.

Au niveau des autres catégories de produits, les résultats montrent que les catégories de produits racines et tubercules, légumineuses et oléagineux, huiles et matières grasses, poissons/crustacés, œufs, sucre, boissons et excitants, conserves et condiments, et les produits non alimentaires sont caractérisées par une demande inélastique,

3. Ces informations sont disponibles sur demande. 
RECHERCHE

Tomgouani LANIE

Tableau 4. Résultats des estimations des élasticités-prix (directe) compensées de la demande

\begin{tabular}{|c|c|c|c|c|c|}
\hline \multirow[t]{2}{*}{ Produits } & \multicolumn{5}{|c|}{ Élasticités-prix de la demande } \\
\hline & National & Urbain & Rural & Pauvres & Non-pauvres \\
\hline \multirow[t]{2}{*}{ Maïs } & $-0.895 * * *$ & $-0.523 * *$ & $-0.907 * * *$ & $-0.972^{* * *}$ & $-0.840 * * *$ \\
\hline & $(0.098)$ & $(0.226)$ & $(0.175)$ & $(0.132)$ & $(0.159)$ \\
\hline \multirow[t]{2}{*}{ Riz } & $-0.350 * * *$ & $-1.052 * * *$ & $-0.278^{*}$ & -0.180 & $-0.524^{* * *}$ \\
\hline & $(0.104)$ & $(0.132)$ & $(0.149)$ & $(0.177)$ & $(0.118)$ \\
\hline \multirow[t]{2}{*}{ Sorgho } & $-2.330 * * *$ & $4.102^{* *}$ & $-3.502 * * *$ & $-3.368 * * *$ & -1.094 \\
\hline & $(0.588)$ & $(1.745)$ & $(0.674)$ & $(0.820)$ & $(0.820)$ \\
\hline \multirow[t]{2}{*}{ Mil } & $-4.403 * * *$ & -0.826 & $-3.869 * * *$ & $-4.823 * * *$ & $-5.224 * * *$ \\
\hline & $(0.904)$ & $(2.370)$ & $(0.966)$ & (0.996) & $(2.058)$ \\
\hline \multirow[t]{2}{*}{ Fonio } & $-39.396 * * *$ & -11.436 & $-50.364 * * *$ & $-42.125^{*}$ & $-55.740 * * *$ \\
\hline & $(13.263)$ & $(20.201)$ & $(18.955)$ & $(23.682)$ & $(13.981)$ \\
\hline \multirow[t]{2}{*}{ Céréales transformées } & $-0.502 * * *$ & $-0.392 * * *$ & $-0.752 * * *$ & $-0.441 * * *$ & $-0.478^{* * *}$ \\
\hline & $(0.086)$ & $(0.118)$ & $(0.138)$ & $(0.147)$ & $(0.110)$ \\
\hline \multirow[t]{2}{*}{ Racines et tubercules } & $-0.249 * * *$ & $-0.408 * * *$ & $-0.215^{* * *}$ & $-0.245 * * *$ & $-0.268 * * *$ \\
\hline & $(0.038)$ & $(0.095)$ & $(0.050)$ & $(0.053)$ & $(0.057)$ \\
\hline \multirow[t]{2}{*}{ Légumineuses et oléagineux } & $-0.736 * * *$ & $-1.106 * * *$ & $-0.737 * * *$ & $-0.807 * * *$ & $-0.728 * * *$ \\
\hline & $(0.065)$ & $(0.113)$ & $(0.093)$ & $(0.096)$ & $(0.094)$ \\
\hline \multirow[t]{2}{*}{ Huiles et matières grasses } & $-0.321 * *$ & -0.315 & -0.340 & -0.269 & $-0.439 * *$ \\
\hline & $(0.158)$ & $(0.206)$ & $(0.228)$ & $(0.250)$ & $(0.203)$ \\
\hline \multirow[t]{2}{*}{ Fruits } & $-1.561 * * *$ & $-1.952 * * *$ & $-2.032 * * *$ & $-2.459 * * *$ & $-1.793 * * *$ \\
\hline & $(0.219)$ & $(0.345)$ & $(0.291)$ & $(0.368)$ & $(0.322)$ \\
\hline \multirow[t]{2}{*}{ Légumes } & $-0.655^{* * *}$ & $-0.407 * * *$ & $-0.729 * * *$ & $-0.767 * * *$ & $-0.539 * * *$ \\
\hline & $(0.061)$ & $(0.112)$ & $(0.077)$ & $(0.085)$ & $(0.092)$ \\
\hline \multirow[t]{2}{*}{ Viandes } & $-1.086 * * *$ & $-0.871 * * *$ & $-1.213 * * *$ & $-1.253 * * *$ & $-0.868 * * *$ \\
\hline & $(0.084)$ & $(0.150)$ & $(0.122)$ & $(0.131)$ & $(0.113)$ \\
\hline \multirow[t]{2}{*}{ Poissons/Crustacés } & $-0.380 * * *$ & $-0.197^{*}$ & $-0.463 * * *$ & $-0.551 * * *$ & $-0.292 * * *$ \\
\hline & $(0.065)$ & $(0.107)$ & $(0.116)$ & $(0.109)$ & $(0.093)$ \\
\hline \multirow[t]{2}{*}{ CEufs } & $-0.967 * *$ & $-1.416 * * *$ & -0.072 & $-2.143 * *$ & $-1.157 * *$ \\
\hline & $(0.410)$ & $(0.473)$ & $(0.973)$ & $(1.065)$ & $(0.458)$ \\
\hline \multirow[t]{2}{*}{ Lait et produits assimilés } & $-1.571 * * *$ & $-2.380 * * *$ & -0.333 & -0.542 & $-1.796 * * *$ \\
\hline & $(0.477)$ & $(0.745)$ & $(0.732)$ & $(0.945)$ & $(0.561)$ \\
\hline \multirow[t]{2}{*}{ Sucre, boissons et excitants } & $-0.200 * * *$ & $-0.460 * * *$ & $-0.129 * *$ & -0.050 & $-0.322 * * *$ \\
\hline & $(0.043)$ & $(0.057)$ & $(0.061)$ & $(0.061)$ & $(0.058)$ \\
\hline \multirow[t]{2}{*}{ Conserves et condiments } & $-0.166 * *$ & $-0.406 * * *$ & -0.221 ** & -0.098 & $-0.271 * * *$ \\
\hline & $(0.065)$ & $(0.095)$ & $(0.097)$ & $(0.103)$ & $(0.085)$ \\
\hline \multirow[t]{2}{*}{ Aliments hors ménage } & 0.104 & $0.808 * * *$ & $-0.324^{*}$ & -0.222 & $0.444 * *$ \\
\hline & $(0.146)$ & $(0.291)$ & $(0.194)$ & $(0.201)$ & $(0.214)$ \\
\hline \multirow[t]{2}{*}{ Autres produits alimentaires } & $-13.350 * * *$ & $-11.052 * * *$ & $-11.793 * *$ & $-26.803 * * *$ & $-8.487 * * *$ \\
\hline & $(2.126)$ & (3.208) & $(5.151)$ & (3.293) & $(2.746)$ \\
\hline \multirow[t]{2}{*}{ Produits non alimentaires } & $-0.240 * * *$ & 0.058 & $-0.358 * * *$ & $-0.392 * * *$ & -0.072 \\
\hline & $(0.088)$ & $(0.120)$ & $(0.140)$ & $(0.129)$ & $(0.114)$ \\
\hline Obs. & 7498 & 2599 & 4899 & 3883 & 3615 \\
\hline
\end{tabular}

Note : les écarts-types robustes sont entre parenthèses. $\left.\left(^{*}\right),{ }^{* *}\right)$ et $\left(^{* * *}\right)$ désignent respectivement les niveaux de significativité de $10 \% ; 5 \%$ et $1 \%$. 
alors que le reste des catégories de produits à savoir : les fruits, les viandes, lait et produits assimilés, et les autres produits alimentaires, sont caractérisées par une demande élastique.

Ces élasticités-prix compensées de la demande varient suivant le milieu de résidence des ménages et leur statut de pauvreté. Suivant le milieu de résidence, nous constatons que la nature de la demande de certains produits se trouve renversée en milieu urbain par rapport au milieu rural. En effet, les produits comme le riz, les légumineuses et oléagineux ainsi que les œufs, caractérisés par une demande élastique en milieu urbain, se révèlent inélastiques en milieu rural. Au contraire, le produit « viandes », caractérisé par une demande élastique en milieu rural, apparaît comme une catégorie de produits à demande inélastique en milieu urbain.

Suivant le statut de pauvreté des ménages, à la différence du milieu de résidence des ménages, les élasticités-prix compensées de la demande des deux catégories de ménages (pauvres et nonpauvres) suivent à quelques exceptions près la tendance nationale. En effet, seul le groupe de produits « viandes » est caractérisé par une demande inélastique chez les ménages non pauvres alors que le groupe de produit lait et produits assimilés semble inélastique chez les ménages pauvres.

Quant aux élasticités-revenus de la demande, le tableau 5 indique qu'au niveau national, parmi les céréales, le maïs, le riz et les céréales transformées ont une élasticité-revenu positive et inférieure à l'unité. Caractérisées par une demande inélastique, ces céréales apparaissent comme des biens de première nécessité.

Pour le reste des céréales (sorgho, mil et fonio), elles présentent une élasticitérevenu de signe négatif indiquant que ce sont des biens inférieurs. En dehors de ces trois dernières céréales, les catégories des produits telles que les œufs, le lait et produits assimilés, les aliments hors ménages et les autres produits alimentaires apparaissent aussi dans la catégorie des biens inférieurs. Quant au reste des produits, mis à part les produits non alimentaires qui apparaissent dans la catégorie des biens de luxe, les autres catégories de produits sont des biens (supérieurs) normaux. Parmi ces biens normaux, les produits alimentaires racines et tubercules, légumineuses et oléagineux, les huiles et matières grasses, les légumes, les poissons et crustacés, le sucre, boissons et excitants, et les conserves et condiments apparaissent dans la catégorie des biens de nécessité. Les élasticités-revenus de la demande varient aussi selon le milieu de résidence des ménages et leur statut de pauvreté mais suivent de façon générale la tendance nationale à la seule exception du produit fruits qui apparaît comme un bien de luxe pour les ménages pauvres.

\section{Simulation de l'impact de la baisse de la TVA sur la pauvreté}

Pour inférer sur l'efficacité de la politique d'allègement fiscal du gouvernement, nous utilisons les élasticités-prix de la demande calculées pour simuler l'impact de cette politique sur le bien-être des ménages et la pauvreté. La méthode utilisée est celle bien connue de la variation compensatoire. Deux types de simulations sont réalisés: dans la première simulation, les ménages ne réagissent pas aux variations des prix (approximation de premier ordre) et dans la seconde simulation, les ménages réagissent aux variations des prix (approximation de second ordre). Nous focalisons notre analyse sur les céréales, en particulier le maïs, le sorgho et le riz qui constituent un axe stratégique en matière de sécurité alimentaire et de réduction de la pauvreté au Togo.

Les résultats des simulations présentés dans le tableau 6 montrent dans 
RECHERCHE

Tomgouani LANIE

Tableau 5. Résultats des estimations des élasticités-dépenses de la demande

\begin{tabular}{|c|c|c|c|c|c|}
\hline \multirow[t]{2}{*}{ Produits } & \multicolumn{5}{|c|}{ Élasticités-revenus de la demande } \\
\hline & National & Urbain & Rural & Pauvres & Non-pauvres \\
\hline \multirow[t]{2}{*}{ Maïs } & $0.330 * * *$ & $0.391 * * *$ & $0.268 * * *$ & $0.270 * * *$ & $0.362 * * *$ \\
\hline & $(0.037)$ & $(0.070)$ & $(0.062)$ & $(0.072)$ & $(0.046)$ \\
\hline \multirow[t]{2}{*}{ Riz } & $0.368 * * *$ & $0.393 * * *$ & $0.171^{*}$ & $0.356 * * *$ & $0.337 * * *$ \\
\hline & $(0.063)$ & $(0.086)$ & $(0.104)$ & $(0.130)$ & $(0.072)$ \\
\hline \multirow[t]{2}{*}{ Sorgho } & $-2.785 * * *$ & -1.127 & $-2.391 * * *$ & $-2.047 * * *$ & $-3.241 * * *$ \\
\hline & $(0.321)$ & $(0.868)$ & $(0.406)$ & $(0.527)$ & $(0.448)$ \\
\hline \multirow[t]{2}{*}{ Mil } & $-6.200 * * *$ & $-7.083 * * *$ & $-5.498 * * *$ & $-5.302 * * *$ & $-6.996 * * *$ \\
\hline & $(0.792)$ & $(1.451)$ & $(0.912)$ & $(0.935)$ & $(1.292)$ \\
\hline \multirow[t]{2}{*}{ Fonio } & $-96.76 * * *$ & 9.744 & $-112.90 * *$ & $-94.26 * * *$ & $-91.59 * * *$ \\
\hline & $(18.137)$ & (9.696) & $(26.035)$ & $(36.082)$ & $(22.020)$ \\
\hline \multirow[t]{2}{*}{ Céréales transformées } & $0.597 * * *$ & $0.613^{* * *}$ & $0.579 * * *$ & $0.589 * * *$ & $0.575 * * *$ \\
\hline & $(0.044)$ & $(0.072)$ & $(0.068)$ & $(0.080)$ & $(0.051)$ \\
\hline \multirow[t]{2}{*}{ Racines et tubercules } & $0.536 * * *$ & $0.412 * * *$ & $0.493 * * *$ & $0.616 * * *$ & $0.504 * * *$ \\
\hline & $(0.040)$ & $(0.082)$ & $(0.058)$ & $(0.072)$ & $(0.058)$ \\
\hline \multirow[t]{2}{*}{ Légumineuses et oléagineux } & $0.300 * * *$ & $0.409 * * *$ & $0.254^{* * *}$ & $0.333^{* * *}$ & $0.229 * * *$ \\
\hline & $(0.051)$ & $(0.074)$ & $(0.078)$ & $(0.083)$ & $(0.072)$ \\
\hline \multirow[t]{2}{*}{ Huiles et matières grasses } & $0.396 * * *$ & $0.420 * * *$ & $0.319 * * *$ & $0.248 * * *$ & $0.406 * * *$ \\
\hline & $(0.053)$ & $(0.062)$ & $(0.075)$ & $(0.085)$ & $(0.065)$ \\
\hline \multirow[t]{2}{*}{ Fruits } & 0.229 & 0.266 & $0.578 * *$ & $1.453 * * *$ & 0.425 \\
\hline & $(0.204)$ & $(0.355)$ & $(0.261)$ & $(0.333)$ & $(0.287)$ \\
\hline \multirow[t]{2}{*}{ Légumes } & $0.488 * * *$ & $0.570 * * *$ & $0.337 * * *$ & $0.387 * * *$ & $0.506 * * *$ \\
\hline & $(0.027)$ & $(0.050)$ & $(0.043)$ & $(0.048)$ & $(0.038)$ \\
\hline \multirow[t]{2}{*}{ Viandes } & $0.510 * * *$ & $0.484^{* * *}$ & $0.260 *$ & $0.313^{* *}$ & $0.574^{* * *}$ \\
\hline & $(0.094)$ & $(0.167)$ & $(0.148)$ & $(0.146)$ & $(0.145)$ \\
\hline \multirow[t]{2}{*}{ Poissons et crustacés } & $0.296 * * *$ & $0.294 * * *$ & $0.184^{* *}$ & $0.195 * *$ & $0.325 * * *$ \\
\hline & $(0.059)$ & $(0.102)$ & $(0.077)$ & $(0.090)$ & $(0.077)$ \\
\hline \multirow[t]{2}{*}{ Eufs } & $-2.209 * * *$ & $-2.636 * * *$ & 0.503 & -0.192 & -1.264 \\
\hline & $(0.695)$ & $(1.029)$ & (1.038) & $(1.478)$ & $(0.856)$ \\
\hline \multirow[t]{2}{*}{ Lait et produits assimilés } & $-1.374 * * *$ & $-1.897 * *$ & -0.953 & 0.278 & $-1.093^{*}$ \\
\hline & $(0.522)$ & $(0.820)$ & $(0.700)$ & $(0.922)$ & $(0.638)$ \\
\hline \multirow[t]{2}{*}{ Sucre, boissons et excitants } & $0.422 * * *$ & $0.601 * * *$ & $0.320 * * *$ & $0.433^{* * *}$ & $0.395 * * *$ \\
\hline & $(0.063)$ & $(0.097)$ & $(0.087)$ & $(0.095)$ & $(0.084)$ \\
\hline \multirow[t]{2}{*}{ Conserves et condiments } & $0.577 * * *$ & $0.508 * * *$ & $0.601 * * *$ & $0.656 * * *$ & $0.562 * * *$ \\
\hline & $(0.034)$ & $(0.050)$ & $(0.048)$ & $(0.072)$ & $(0.035)$ \\
\hline \multirow[t]{2}{*}{ Aliments hors ménages } & $-0.274^{*}$ & $-0.979 * * *$ & 0.240 & $0.494^{* * *}$ & $-0.516^{* *}$ \\
\hline & $(0.143)$ & $(0.360)$ & $(0.168)$ & $(0.192)$ & $(0.204)$ \\
\hline \multirow[t]{2}{*}{ Autres produits alimentaires } & $-7.71 * * *$ & -0.064 & $-21.95 * * *$ & -6.541 & $-6.209 * *$ \\
\hline & $(2.429)$ & (3.313) & $(4.855)$ & $(4.981)$ & $(2.691)$ \\
\hline \multirow[t]{2}{*}{ Produits non alimentaires } & $2.361 * * *$ & 0.058 & $2.390 * * *$ & $2.176 * * *$ & $2.415^{* * *}$ \\
\hline & $(0.045)$ & $(0.120)$ & $(0.060)$ & $(0.090)$ & $(0.055)$ \\
\hline Obs. & 7498 & 2599 & 4899 & 3883 & 3615 \\
\hline
\end{tabular}

Note : les écarts-types robustes sont entre parenthèses. $\left({ }^{*}\right),\left({ }^{* *}\right)$ et $\left({ }^{* * *}\right)$ désignent respectivement les niveaux de significativité de $10 \% ; 5 \%$ et $1 \%$. 


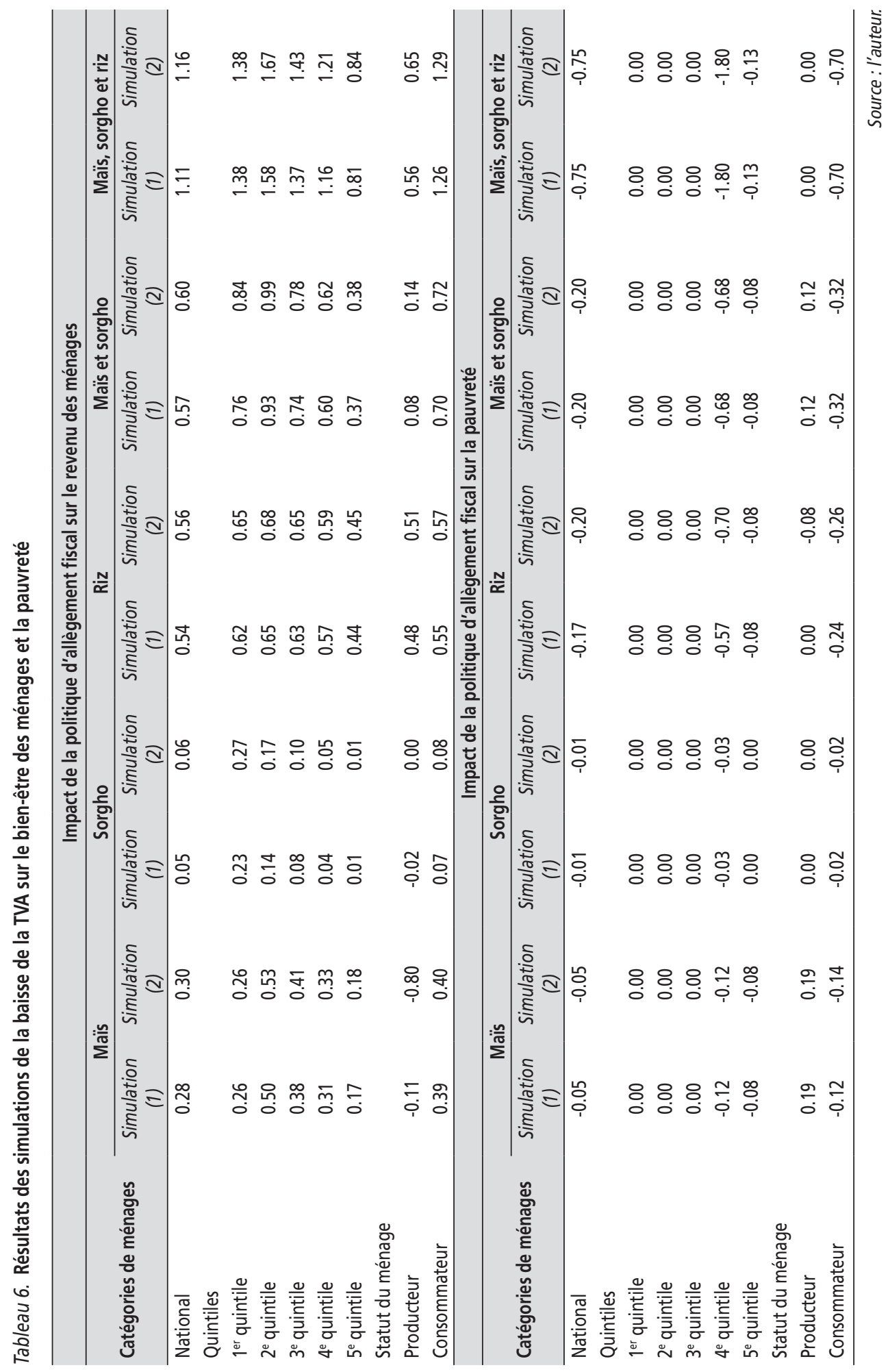


l'ensemble que l'impact de la politique d'allègement fiscal est sous-estimé lorsque la réponse des ménages n'est pas prise en compte. Cette politique d'allègement fiscal aurait eu un impact positif sur le revenu des ménages quelle que soit la spéculation considérée au niveau national et suivant les différentes catégories de ménages (à l'exception des producteurs de maïs et du sorgho) mais l'impact est marginal même s'il devient plus important lorsque les trois spéculations sont prises ensemble. Cet impact positif s'est traduit par une baisse de la pauvreté. Les ménages consommateurs purs qui auraient bénéficié plus de la baisse de la TVA que les ménages producteurs ont connu une diminution de leur pauvreté alors que la pauvreté est restée inchangée au niveau des ménages producteurs (elle aurait même augmenté parmi les ménages producteurs de maïs). Au contraire, les ménages les plus pauvres auraient bénéficié plus de la baisse de la TVA que les ménages plus aisés mais cela ne s'est pas traduit par une baisse de la pauvreté au niveau de ces catégories de ménages, cette politique aurait permis à seulement certains ménages plus aisés de sortir de la pauvreté.

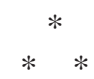

Dans cet article, nous avons estimé les élasticités-prix et revenu de la demande des produits alimentaires et simulé l'impact de la politique d'allègement fiscal sur le bien-être des ménages et la pauvreté au Togo. Les résultats montrent que les élasticités-prix compensées de la demande ont des signes conformes à la théorie économique. Les élasticités-revenus indiquent que les céréales comme le maïs, le riz et les céréales transformées sont des biens de nécessité alors que le mil, le sorgho et le fonio sont des biens inférieurs. Ces élasticités-prix et revenus de la demande varient selon le milieu de résidence des ménages et leur statut de pauvreté.

Quant aux résultats des simulations, ils montrent effectivement que l'impact de la baisse de la TVA est sous-estimé lorsque la réponse des ménages n'est pas prise en compte. Cette politique d'allègement fiscal aurait eu un impact positif sur le bien-être des ménages au niveau national et suivant les différentes catégories de ménages, et se serait traduite par une baisse de la pauvreté seulement au niveau national, parmi les ménages plus aisés ainsi qu'au niveau des ménages consommateurs. Toutefois, cet impact positif reste marginal. Il suggère au niveau des céréales considérées que pour lutter efficacement contre la cherté de la vie et réduire la pauvreté, le Gouvernement devrait mettre l'accent sur les biens de nécessité qui ont une demande élastique. 


\section{RÉFÉRENCES BIBLIOGRAPHIQUES}

Abramovsky L., Attanasio O., Phillips D. (2011). Demand responses to changes in consumer prices in Mexico: lessons for policy and an application to the 2010 Mexican tax reforms. Working Paper, June, pp. 1-27.

Agbodji A. E., Amouzouvi K., Bouare K., Diagne I., Kielem A. (2013). Analyse de l'Impact de la Hausse Mondiale des Prix et des Politiques de réponse du gouvernement sur la pauvreté. PEP Working Paper, pp. 1-52.

Banks J., Blundell R., Lewbel A. (1997). Quadratic Engel Curves and Consumer Demand. The Review of Economics and Statistics, vol. 79, n 4, pp. 527-539.

Blundell R., Robin J. M. (1999). Estimation in Large and Disaggregated Demand Systems: An Estimator for Conditionally Linear Systems. Journal of Applied Econometrics, vol. 14, n 3, pp. 209-232.

Brown A., Deaton A. (1972). Surveys in Applied Economics: Models of Consumer Behaviour. The Economic Journal, vol. 82, n 328, pp. 1145-1236.

Capéau B., Dercon S. (1998). Prices, local measurement units and subsistence consumption in rural surveys: an econometric approach with an application to Ethiopia. Working Paper, 1987, pp. 1-36.

Deaton A., Muellbauer J. (1980). An Almost Ideal Demand System. The American Economic Review, vol. 70, n 3, pp. 312-326.

Deaton A. (1986). Demand analysis. Handbook of Econometrics, vol. III, pp. 1768-1829.

Deaton A. (1987). Estimation of Own and Cross Price Elasticities from households survey Data. Journal of Econometrics, n 36, pp. 7-30.

Deaton A. (1988). Quality, Quantity, and Spatial Variation of Price. The American Economic Review, vol. 78, n³, pp. 418-430.

Deaton A. (1989). In Rice Prices and Income Distribution in Thailand: A Non-parametric Analysis. The Economic Journal, ${ }^{\circ} 99$, Conference1989, pp. 1-37.
Deaton A. (1990). Price Elasticities from Survey Data: Extensions and Indonesian Results. Journal of Econometrics, $\mathrm{n}^{\circ} 44$, pp. 281-309.

Deaton A. (1997). The Analysis of Household Surveys: A Microeconometric Approach to Development Policy. The Johns, Baltimore and London, The World Bank.

Deaton A., Zaidi S. (2002). Guidelines for Constructing Consumption Aggregates For Welfare Analysis. The World Bank, Washington D.C.

Deaton A., Dupriez O. (2011). Using Unitvalues to Assess Spatial Price Differences: Evidence from India and Brazil. 5th Technical Advisory Group Meeting, April 2011, Washington D.C.

Gibson J., Rozelle S. (2005). Prices and Unit Values in Poverty Measurement and Tax Reforms Analysis. Working Paper, pp. 1-45.

Gibson, J. (2013). The crisis in food price data. Global Food Security, 2, 97-103.

Mckelvey C. (2011). Price, unit value, and quality demanded. Journal of Development Economics, n ${ }^{\circ}$ 95, pp. 157-169. doi:10.1016/ j.jdeveco.2010.05.004

Nzuma J.-M., Sarker R. (2010). An error corrected almost ideal demand system for major cereals in Kenya. Agricultural Economics, $n^{\circ} 41$, pp. 43-50.

Poi B. P. (2008). Demand-system estimation: Update. The Stata Journal, vol. 8, $\mathrm{n}^{\circ} 4$, pp. 554-556.

Questionnaire des indicateurs de base du bienêtre (Quibb) (2006). Manuel de l'enquêteur. Direction de la Stastistique et de la Comptabilité nationale, Juin 2006.

Shonkwiler S. J., Yen S. (1999). Two-step Estimation of a Censored System of Equations. American Journal of Agricultural Economics, vol. 81, n ${ }^{\circ}$, pp. 972-982.

Tafere K., Taffesse A. S., Tamiru S. (2010). Food Demand Elasticities in Ethiopia: Estimates Using Household Income 
RECHERCHE

Tomgouani LANIE

Consumption Expenditure (HICE) Survey Data. ESSP2 Discussion Paper 011, pp. 1-53.

Tchabletienne K., Koffi-Tessio E. M., Diagne A. (2010). Demande du riz importé, demande du riz produit localement au Togo: une estimation à partir du modèle Almost Ideal Demand System (AIDS). Poster presented at the 3rd African Association of Agricultural
Economists (AAAE), Conference, Cape Town, South Africa, September pp. 19-23.

Yen S. T., Biing-Hawan L., Smallwood D. M. (2003). Quasi and simulated likelihood approaches to censored demand system: food consumption by food stamp recipient in the United States. American Journal of Agricultural Economics, ${ }^{\circ}$ 85, pp. 458-478. 